DOI: https://doi.org/10.11144/Javeriana.upsy18-4.ndic

\title{
Neoliberalismo y declive institucional en Chile. La individualización del malestar laboral en psicólogos*
}

Neoliberalism and Institutional Decline in Chile: Labor Unrest Individualization in Psychologists

Recepción: 09 Junio 2019 | Aceptación: 11 Octubre 2019

\author{
Tania de Armas Pedraza ${ }^{a}$ \\ Universidad de Playa Ancha, Chile \\ ORCID: http://orcid.org/0000-0002-3293-0128 \\ Cristian Venegas Ahumada \\ Universidad de Playa Ancha, Chile \\ ORCID: http://orcid.org/0000-0001-5875-3767 \\ Gonzalo Salas \\ Universidad Católica del Maule, Chile \\ ORCID: http://orcid.org/0000-0003-0707-8188 \\ María Constanza Aguilar- \\ Bustamante \\ Universidad Santo Tomás, Colombia \\ ORCID: http://orcid.org/0000-0003-2990-9667
} a Autor de correspondencia. Correo electrónico:
tania.dearmas@upla.cl

Para citar este artículo: De Armas Pedraza, T., Venegas Ahumada, C., Salas, G., \& Aguilar-Bustamante, M. C. (2019). Neoliberalismo y declive institucional en Chile. La individualización del malestar laboral en psicólogos. Universitas Psychologica, 18(4), 1-17. ht tps://doi.org/10.11144/Javeriana.upsy18-4.ndic

\section{RESUMEN}

El objetivo del estudio fue describir la experiencia del malestar laboralnarrada por psicólogos chilenos en grupos Facebook relacionados con la formación y ejercicio profesional de la psicología. Se realizó un estudio cualitativo aplicado a tres grupos, mediante el software NVivo 11 y orientado por un análisis sociológico de contenido, complementado con datos cuantitativos. Los hallazgos indican que el neoliberalismo produce descolectivización, que se asocia a la crisis de representación del Colegio de Psicólogos de Chile (COLEPSI), por cuanto existe una escasa legitimidad organizacional expresada en la merma de la legitimidad moral. El $25 \%$ de los comentarios señalan que el COLEPSI no se pronuncia sobre la precariedad laboral ni la desregulación de la oferta de pregrado (22\%). Finalmente, frente al declive institucional, los psicólogos deben asumir la tramitación de su malestar laboral, específicamente, mediante la publicación en diversos grupos Facebook que poseen potencialidad de articulación política para generar cambios.

\section{Palabras clave}

psicólogos; neoliberalismo; individualización; malestar laboral; legitimidad organizacional; Colegio de Psicólogos de Chile.

\footnotetext{
ABSTRACT

The objective of this study was to describe labor unrest experience as recounted by Chilean psychologists in Facebook groups related to Psychology professional practice and education. A qualitative research was applied to 3 groups using Nvivo 11 software, guided by sociological content analysis and complemented with quantitative data.
} 
Findings indicate that neoliberalism produces decollectivisation, which is associated to the representative crisis of College of Psychologists of Chile (COLEPSI), given there is scant organizational legitimacy expressed in a lack of moral legitimacy. $25 \%$ of comments claim that COLEPSI does not declare against job insecurity nor undergraduate course-offer lack of regulation (22\%). Finally, facing institutional decline psychologists must take on their labor unrest processing, especially by publishing in different Facebook groups with a potential for political articulation in order to generate changes.

Keywords

psychologists; neoliberalism; individualization; labor unrest; organizational legitimacy; College of Psychologists of Chile.

El trabajo y el empleo profesional de los psicólogos es una temática que requiere una diversidad de análisis para lograr una comprensión cabal de fenómenos como la satisfacción laboral (De Souza \& De la Coleta, 2015; Idsoe, 2006; Maeran, Robusto, \& Anselmi, 2015), la cual afecta a todas las profesiones y, entre estas, a la psicología. Este tipo de temáticas han sido escasamente estudiadas por los psicólogos de la salud ocupacional (p. ej., Sauter \& Hurrell, 2017), más aún, si se considera que la literatura documenta que los psicólogos y profesionales de la salud mental tienden a descuidar su propia salud (Dattilio, 2015). Este tipo de estudios colindan con traumas y experiencias extremas (Johnson et al., 2011), burnout (Roncalli \& Byrne, 2016) y humor laboral (Malinowski, 2013). Por otro lado, desde la psicología de las organizaciones, se han encontrado dos factores que tradicionalmente se han considerado como indicadores del trabajo y el empleo, estos son: la satisfacción laboral de los empleados y un grado óptimo de estrés de rol (Gamboa, Gracia, Ripoll, \& Peiró, 2007). En este sentido, se evidencian dos posturas frente al primer factor; una, quienes afirman que la satisfacción en el trabajo podría concebirse como una consecuencia; si alguien es empleable, es capaz de encontrar empleo y además, lograr uno satisfactorio y adecuado (Forrier \& Sels, 2003). Respecto al segundo factor, se observa que la satisfacción en el trabajo se encuentra relacionada con indicadores como: el estatus (empleado, desempleado, estudiante, etc.), los ingresos, el salario, el cargo que se ocupa, el nivel jerárquico en la organización, el tipo de contrato y las posibilidades de promoción (Hillage \& Pollard, 1998). Por su parte, diversos autores, relacionan la satisfacción y la calidad del empleo, con aspectos como la sincronía entre el trabajo, el título profesional y las perspectivas de crecimiento personal (Aguilar-Bustamante, 2011; Gamboa et al., 2007; Rentería \& Malvezzi, 2008), si la ocupación coincide con la profesión, que es el caso que ocupa este trabajo.

El presente artículo destaca los fundamentos del diseño sociopolítico y económico neoliberal y los resultados en términos de producción que afectan la sostenibilidad del bienestar, desempeño y malestar laboral (Peiró, Ayala, Tordera, Lorente, \& Rodríguez, 2014; Sharrocks, 2014) de los psicólogos ${ }^{1}$ en Chile, lo cual será interpretado desde el marco sociológico donde se sitúa.

En el año 1980, se aprueba la entonces nueva Constitución Política del Estado de Chile que institucionaliza las bases del modelo sociopolítico y económico neoliberal (Friedman \& Friedman, 1986) que consagra la libertad de enseñanza y de empleodejando amparada la defensa de derechos individuales, para así proteger la libertad económica que es definida como un derecho fundamental que confiere a los individuos la más amplia voluntad para trabajar, producir, consumir e invertir de cualquier forma que lo deseen (Heritage Foundation, 2013; Kaiser, 2017).

La Constitución chilena de 1980 ha consagrado la defensa de la "libertad de enseñanza" y la "libertad de empleo", y sus efectos han sido letales en cuanto a la formación de pregrado y ejercicio profesional de la psicología (De Armas, Ramos, Cárdenas, Ferretti, \& Venegas, 2017; De Armas, Ramos, \& Venegas, 2016a, 2016b; De Armas, Ramos, Venegas, \& Lorenzo, 2016; Venegas, 2010, 2013, 2014, 2019a, 2019b). Primero, las universidades privadas chilenas concentran el $88 \%$ de la oferta de pregrado en psicología y las universidades estatales alcanzan solo un $12 \%$. Segundo, el aumento en el número de programas de pregrado en psicología, así, en el año 1982 existían 
siete programas y el año 2016 un total de 144 programas, por tanto, un incremento de $1954 \%$. Tercero, en el período (1996-2017) como consecuencia de la multiplicación de los programas de pregrado, el número de titulados ha experimentado un crecimiento del $669 \%$. Cuarto, el trabajo no decente (precariedad laboral/ flexibilidad laboral) alcanza a un $64.5 \%$ de los psicólogos que ejercen la profesión. En una situación como esta, la formación y el ejercicio profesional en Psicología han avanzado hacia un marcado proceso de desprofesionalización. Quinto, emerge el malestar laboral (Blanch, 2007; Venegas, 2013) como resultado de un modelo sociopolítico y económico neoliberal que precariza el empleo.

El diseño sociopolítico y económico neoliberal (p. ej., De Armas \& Venegas, 2015) promueve la descolectivización (Báez, 2017) al tener como un bien jurídicamente protegido los derechos individuales. Un ejemplo que marca un hito en la descolectivización para las profesiones fue la promulgación el 7 de febrero de 1981 de la Ley 3621 que fija normas sobre Colegios Profesionales (Salas \& Lizama, 2013). Esta legislación transformó a los Colegios Profesionales en Asociaciones Gremiales (AG) y dispone, entre otros aspectos, que no será prerrequisito estar inscrito en el colegio profesional para ejercer legalmente la profesión y, además, restringe la jurisdicción ética solo a quienes se encuentren afiliados al Colegio o acepten voluntariamente su jurisdicción ética. Finalmente, carece de atribuciones para revocar el ejercicio profesional a quienes infrinjan de modo grave el código de ética profesional (De Armas, Ramos, \& Venegas, 2016a, 2016b).

En América Latina, las organizaciones gremiales cumplen diversos papeles y en algunos países estas juegan un papel que busca mejorar las condiciones y la calidad de la formación y del ejercicio profesional, así, se pueden encontrar dos tipos de organizaciones que tienen a cargo la psicología como disciplina y profesión. Las primeras, corresponden a las agrupaciones que reúnen la formación de la psicología, que se llevan a cabo en instituciones de educación superior (IES), a través de programas de pregrado y postgrado (Aguilar-Bustamente, 2017).

El segundo tipo de organización hace referencia a las asociaciones profesionales que se encuentran en cada país (local), regional (continental) e internacional (global). Sus funciones están alrededor de los siguientes tópicos: potenciar la promoción y el desarrollo técnico y científico de la profesión; velar por la actuación profesional de acuerdo con las normas contenidas en los respectivos códigos deontológicos del psicólogo de cada país; promocionar y fortalecer las áreas de aplicación de la psicología, entre muchas otras que surgen en las dinámicas particulares de cada país (AguilarBustamante, 2017).

Para el caso chileno, una consecuencia directa de la descolectivización (Báez, 2017) es que el número de afiliados al Colegio de Psicólogos de Chile (COLEPSI), tomando como base el número de titulados en el período (1996-2016) llega aproximadamente a un $10 \%$, lo cual puede asociarse a una baja legitimidad organizacional ( Suchman, 1995). Además, solo un $20 \%$ de los afiliados al COLEPSI mantiene sus cuotas sociales o membrecía al día (Melús, 2017) 2 , fenómeno asociado a una baja legitimidad pragmática (Suchman, 1995). De este modo, la AG se encuentra en una posición poco favorable como organización para representar los temas que resultan de interés para los psicólogos en general, mantener en el tiempo a sus afiliados y motivar la inscripción en el Colegio.

Como consecuencia del diseño sociopolítico institucional neoliberal, los cambios sociales acaecidos y en curso han desplazado la centralidad de un amplio espectro de la institucionalidad vigente (Dubet, 2006). Siguiendo el planteamiento del declive institucional, resulta lógico sostener que la experiencia del malestar laboral (Blanch, 2007; Venegas, 2013) de los psicólogos en su ejercicio profesional está siendo canalizado por vías no institucionales, es decir, se verifica una desinstitucionalización con la consiguiente individualización del malestar.Por lo mismo, uno de los soportes(Martuccelli, 2007a, 2007b, 2010a, 2010b) de la experiencia del malestar 
laboral lo constituyen las redes sociales, especialmente grupos Facebook creados por psicólogos, donde se comparten temas de interés vinculados a la formación y ejercicio profesional.

En este sentido, se ha estudiado la relación entre el shock macroeconómico de la inestabilidad laboral y el bienestar psicológico, analizándose más de 1.2 billones de mensajes de Twitter (Proserpio, Counts, \& Jain, 2016). En la presente investigación, se describen los temas emergentes vinculados a las experiencias del malestar laboral que son publicadas como comentarios en los grupos Facebook que han sido definidos por sus administradores como "públicos", ya que el propósito de este artículo es describir la experiencia del malestar laboral en psicólogos cuya experiencia se encuentra invisibilizada y excluida del discurso oficial del COLEPSI. Esta organización tiene entre sus objetivos: "Promover y velar por la racionalización, desarrollo, protección, perfeccionamiento, progreso y prerrogativas de la profesión de Psicólogo y por el regular y correcto ejercicio de la profesión por parte de sus asociados" (Colegio de Psicólogos de Chile, 2018, p. 1). Si se considera de este modo, este trabajo describe fragmentos de los discursos del malestar en psicólogos en relación con el discurso del COLEPSI. Este aspecto resulta concordante con el objetivo general de la presente investigación, vinculado a describir la experiencia del malestar laboralnarrada por psicólogos chilenos en grupos Facebook relacionados con la formación y ejercicio profesional de la psicología.

\section{El Neoliberalismo y el declive institucional del Colegio de Psicólogos de Chile: la individualización del malestar laboral}

En el diseño sociopolítico económico neoliberal, el rol del Estado se reduce a políticas públicas focalizadas que dejan espacios más amplios de la vida social entregadas a la volatilidad del mercado con su ley de la oferta y la demanda, produciéndose una reestructuración laboral marcada por el desempleo, la precarización y la desaparición de derechos sociales, es decir, una verdadera crisis del empleo. En este contexto, los individuos siguen trayectorias laborales inestables marcadas por la incertidumbre(Andrade, 2014; Castel, 1997, 2004; Dubar, 2001, 2002; Friedman \& Friedman, 1986; Soto, 2011).

En la sociedad neoliberal chilena, se enfrenta la prueba del mérito(Araujo \& Martuccelli, 2012a, 2012b; Gómez, 2010), donde los trabajadores deben responder y así demostrar capacidad para el trabajo, eficiencia y rendimiento. Esta respuesta frente al desafío se hace al considerar como referente un ideal(Araujo, 2009) de proactividad y competencia en un contexto caracterizado como "crisis del empleo".

Ingresar al mundo del trabajo, corresponde a la inserción laboral y mantenerse, cambiarse o moverse a un mejor trabajo dentro o fuera de la empresa se refiere a la empleabilidad (Aguilar-Bustamante, 2011; Gamboa et al., 2007). Esta empleabilidad se expresa como una respuestaespecífica por parte de los individuos, debiendo recurrir para ello a determinados soportes, conceptualizados como:

[...] conjuntos heterogéneos de elementos, reales o imaginarios, que se despliegan a través de un entramado de vínculos, que suponen un diferencial de implicación según las situaciones y las prácticas, y gracias a los cuales, los individuos se sostienen, porque están sostenidos, y están sostenidos, porque se sostienen, en medio de la vida social. (Martuccelli, 2007b, pp. 81-82)

Las redes sociales virtuales sirven de soporte para los individuos que, en una sociedad como la chilena, se trasforma en un espacio para la tramitación del malestar laboral (Martuccelli, 2010b), verificándose una forma de individualización contemporánea, entendida como "compulsión de encontrar y buscar nuevas certezas para uno mismo y para quienes carecen de ellas. Pero también significa nuevas interdependencias" (Robles, 1999, p. 293). Estas formas de certeza y estabilidad son buscadas, generando relaciones mediante las redes sociales virtuales. 
Al analizar en Facebook la experiencia laboral de los psicólogos, se percibe cómo se sostienen desde ese espacio virtual, la tramitación del malestar laboral que les aqueja. En este sentido, se entiende por malestar laboral la experiencia de los psicólogos expresada de modo cognitivo, afectivo, emocional, en términos de disconformidad con respecto al ejercicio de la profesión, el cual posee una interrelación entre tres dimensiones: institucional, referida al sistema económico neoliberal; condiciones laborales, es decir, remuneración, tipo de contrato y organización del trabajo y una dimensión organizacional, referida a la legitimidad organizacional del COLEPSI (Blanch, 2007; Venegas, 2013), la que se conceptualiza como "una percepción generalizada de que las acciones de una organización son deseables, adecuadas o apropiadas dentro de un sistema socialmente construido con normas, valores, creencias y definiciones" (Suchman, 1995, p. 574). Para sistematizar la relevancia que tiene la legitimidad para las organizaciones se sostienen los siguientes puntos:

1. Las organizaciones con más legitimidad tienen acceso a un mayor número de recursos.

2. Las organizaciones con más legitimidad sobreviven en mayor medida que aquellas que poseen menos legitimidad.

3. Las organizaciones ganan legitimidad cuando demuestran y comparten los intereses (objetivos) de sus grupos de interés.

4. Las organizaciones ganan legitimidad cuando demuestran y comparten los valores y creencias con sus grupos de interés.

5. Las organizaciones ganan legitimidad cuando se comprometen y desarrollan métodos, prácticas, ideas, etc. que están ampliamente aceptadas por profesionales y científicos del sector.

6. El coste de una organización para ganar legitimidad pragmática es menor que la de adquirir legitimidad moral y a su vez menor que adquirir legitimidad cognitiva (Diez, Blanco, \& Prado, 2010).

En la presidencia del directorio del COLEPSI encabezada por Leonardo Villarroel (2009-2011), se creó el Reglamento de Filiales y el Departamento de Filiales que entregó un soporte reglamentario y administrativo a partir del cual, en el período posterior, el Presidente, Rodrigo Molina (2012-2015), potenció dicho departamento a través de la inversión de recursos que posibilitó tener siete filiales activas ${ }^{3} \mathrm{y}$ se dispuso un cambio en la normativa que permitió que las regionales tuvieran más recursos financieros, esto es, que el $70 \%$ del monto de las cuotas sociales de los afiliados a las filiales regionales quedarán destinados a la propia filial y solo el $30 \%$ se traspasará a la administración del Directorio Nacional del COLEPSI ${ }^{4}$. Además, se creó el "Consejo de Representantes Regionales" que sesionaba de modo anual como una forma de cuenta pública hacia el Directorio Nacional (R. Molina, comunicación personal, 14 de abril 2018) ${ }^{5}$, realizando un importante esfuerzo por potenciar la regionalización.

Ha existido conciencia en los directorios del COLEPSI sobre la necesidad de avanzar en el fortalecimiento organizacional, elevar la participación de sus afiliados, aumentar el posicionamiento e influencia de la profesión en los debates nacionales en torno a políticas de salud mental, como en distintas áreas donde la psicología podría aportar a la sociedad (Acuña, 2017; Melús, 2016, 2017).

Sin embargo, como se ha mencionado previamente, el diseño sociopolítico institucional neoliberal (p. ej., De Armas \& Venegas, 2015) a través de la Constitución Política del Estado (CPE) ha generado descolectivización (Báez, 2017), por cuanto la Carta Fundamental defiende derechos individuales, más no derechos colectivos ( Atria, 2015; Atria, Larraín, Benavente, Couso, \& Joignant, 2013). A lo señalado en términos de descolectivización, es posible agregar que la transformación del COLEPSI en una A $G$ de afiliación voluntaria, jurisdicción ética restringida solo a quienes están afiliados, no presenta potestades para 
regular ampliamente el ejercicio profesional (De Armas, Ramos, \& Venegas, 2016b). Finalmente, la CPE defiende la concepción de la teoría económica neoliberal (Friedman \& Friedman, 1986), haciendo que el Estado no tenga atribuciones para limitar la cantidad de programas de pregrado para estudiar psicología (libertad de enseñanza) ni puede establecer como prerrequisito para el ejercicio legal de la profesión de la Psicología afiliarse al COLEPSI (libertad de empleo y asociación). Una situación como la descrita evidencia las características propias de un Estado subsidiario (Dávila, 1998) que permite entender que la crisis de representación por parte del COLEPSI está influida por elementos socioestructurales y macropolíticos, vinculados a la falta de representatividad y legitimidad del orden sociopolítico institucional neoliberal, para traducir las demandas de la ciudadanía por el respeto de los derechos sociales en una realidad (Atria, 2015; Atria et al., 2013; Garretón, 2016). Este es el declive institucional (Dubet, 2006) que afecta la legitimidad organizacional (Suchman, 1995) del COLEPSI y de los Colegios Profesionales en general ${ }^{6}$. Esto explica, por lo tanto, que el malestar de los psicólogos sea tramitado mediante otras organizaciones.

Entre dichas organizaciones merece una especial mención el grupo Facebook Asociación de Psicólogos Chilenos (APSICH), anteriormente denominada Psicólogos Disconformes. Este grupo ha venido actuando como minoría activa (Asún, 2002; Moscovici, 1985) que no solo ha permitido tramitar el malestar laboral como espacio virtual de soporte (Martuccelli, 2007b), sino, además, avanzan hacia una politización del malestar, propiciando la capitalización simbólica de ser un referente genuino frente a lo que denominan una crisis de representación del COLEPSI, para de este modo construir su legitimidad organizacional (Suchman, 1995), a tal punto que después de un período de articulación informal como grupo Facebook, actualmente APSICH ha obtenido la personería jurídica.

$\mathrm{Al}$ interior de aquellas iniciativas de APSICH, se encuentran: "Semáforo salarial de empleadores", la "Lista negra de empleadores", diversos encuentros presenciales donde se han dado cita psicólogos para conversar los temas que les preocupan, reflexiones publicadas en su Facebook sobre asuntos de contingencia que atañen a la psicología en Chile, "avisos de utilidad pública", "autocuidado para psicólogos", incluso la publicación de una carta abierta que denuncia la precarización de la psicología (Cahís, 2016). Para complementar, APSICH avanza en el establecimiento de distintos nodos Norte, Centro y Sur de la geografía nacional, para descentralizar y tener una actividad zonal más pertinente.

A la APSICH es necesario agregar los grupos "Psicólogos chilenos por la regulación de los sueldos" y "Movimiento de psicólogos chilenos" que han impulsado una serie de reuniones entre psicólogos, y marchas para visibilizar especialmente la precariedad laboral. En este mismo sentido, en las ciudades de Coquimbo y La Serena, se ha creado el grupo de psicólogos liderados por Luis Poblete, conectados por un grupo de WhatsApp que se reúnen periódicamente desde el año 2018 para analizar diversas cuestiones referentes a la psicología como profesión.

Para finalizar este apartado, existe una transversalidad de temáticas entre los distintos grupos Facebook lo que da cuenta de una crisis de legitimidad organizacional (Suchman, 1995) del COLEPSI, asociada a elementos socioestructurales y macropolíticos, que limita de modo importante la capacidad de la $A G$ para conducir o liderar el cambio en aquellas temáticas que causan el malestar en los psicólogos.

\section{Método}

\section{Diseño}

Se trata de una investigación exploratoria (Hernández, Fernández, \& Baptista, 2010) con un diseño cualitativo de estudio de caso instrumental (Stake, 1999), es decir, se emplea el caso para describir un proceso más amplio como es el malestar laboral asociado a las transformaciones producidas en el ámbito de la 
formación y ejercicio profesional de la psicología en Chile.

\section{Muestra}

Se realizó un muestreo intencionado (Salinas, 2010) respecto a aquellos grupos públicos 7 de Facebook de psicólogos cuya descripción del grupo se relaciona con la formación y/o ejercicio profesional de la psicología. Los tres grupos Facebook que en el contexto chileno abordan estas temáticas estudiadas se describen en la Tabla 1. Se integraron a la muestra, para el posterior análisis, un total de 147 comentarios.

\section{Tabla 1}

Información de los grupos Facebook de psicólogos

\begin{tabular}{|c|c|c|c|}
\hline $\begin{array}{c}\text { Nombre del grupo } \\
\text { Facebook }\end{array}$ & $\begin{array}{c}\text { Descripción del grupo } \\
\text { Facebook }\end{array}$ & $\begin{array}{l}\text { Número de } \\
\text { miembros o } \\
\text { seguidores }\end{array}$ & $\begin{array}{l}\text { Condición } \\
\text { del grupo }\end{array}$ \\
\hline $\begin{array}{l}\text { Colegio de Psicólogos } \\
\text { de Chile }\end{array}$ & $\begin{array}{l}\text { Organización fundada para el } \\
\text { cuidado del ejercicio } \\
\text { profesional de la Psicologia } \\
\text { en Chile }\end{array}$ & 22540 & Público \\
\hline $\begin{array}{l}\text { Psicólogos Chilenos por } \\
\text { la regulación de los } \\
\text { sueldos }\end{array}$ & Sin información. & 3272 & Público \\
\hline $\begin{array}{l}\text { Movimiento de } \\
\text { Psicólogos Chilenos }\end{array}$ & $\begin{array}{l}\text { Es grupo de psicólogos y } \\
\text { psicólogas que nos unimos en } \\
\text { torno a un diagnóstico común: } \\
\text { la mayoría de los psicólogos y } \\
\text { psicólogas accede a } \\
\text { condiciones laborales } \\
\text { precarias. }\end{array}$ & 5559 & Público \\
\hline
\end{tabular}

Una vez seleccionados estos grupos se escogió el corpus de datos cualitativos integrado por el total de comentarios relacionados con las "condiciones laborales" publicados por los psicólogos en "grupos públicos" de Facebook.

\section{Análisis de datos}

El método de análisis empleado al corpus de comentarios fue el análisis sociológico de contenido(Alonso, 1998; Cáceres, 2008; Flick, 2004, 2015; Gibbs, 2012). Se ocupó el softwareNVivo 11 para el procesamiento de la información (QSR Internacional, 2017). Se utilizó específicamente Ncapture con la finalidad de documentar los comentarios efectuados en los grupos Facebook y así construir una "matriz de adyacencia" para formar la representación gráfica (QSR International, 2017, 2018), siguiendo como modelos una diversidad de estudios ( $\mathrm{p}$. ej., Firat, 2017; Mustaffa \& Ibrahim, 2014; Ramadan, 2017; Sagun \& Luyt 2017). Los pasos realizados fueron los siguientes:

1. Se utilizó la herramienta Ncapture mediante la cual se recopilaron todos los comentarios y publicaciones efectuadas en los grupos Facebook identificados.

2. Se utilizó la herramienta árbol de palabras para mostrar la forma en que aparecía el contenido cualitativo asociado a las "condiciones laborales" para el ejercicio de la psicología, llegando a establecerse que se trataba de un tema en torno al cual se organizaban de manera central los significados importantes para el objeto de estudio.

3. Se realizó una consulta mediante la herramienta búsqueda de texto para la expresión "condiciones laborales", marcando la opción que especifica "buscar con sinónimos". De este modo se redujo el material cualitativo en función de los objetivos de estudio. También se optó por dejar fuera del análisis las ofertas de trabajo publicadas, por cuanto no se refieren a la experiencia de los psicólogos.

4. El análisis de los comentarios en los grupos Facebook se inició con categorías a priori, es decir, los nodos se crearon bajo el enfoque top-down (Gutiérrez \& Palacios 2016). En una primera etapa, se utilizaron las categorías de sistema económico neoliberal (De Armas \& Venegas, 2015), precariedad laboral y malestar laboral (Venegas, 2013).

5. Se fueron creando subnodos que permitieron aumentar en la singularidad y sensibilidad del proceso de categorización para dar cuenta de la profundidad cualitativa de los datos. En este punto, se opera con una estrategia bottom-up (Gutiérrez \& Palacios, 2016). Esto ha llevado en algunos casos a fusionar subnodos, agrupando en un nuevo nodo el contenido cualitativo de los comentarios. 
6. Si se considera el número de comentarios por cada nodo, se procedió a efectuar gráficos mediante el programa Excel, para describir primero, temas principales comentados por los psicólogos de modo transversal a los grupos, los cuales son comentados específicamente en el grupo Facebook del COLEPSI, y finalmente se grafica una comparación de temas entre los diversos grupos Facebook para interpretar dichos resultados con base en el marco teórico utilizado.

\section{Consideraciones éticas}

La información extraída considera el anonimato de los participantes, dado que en ningún momento se expone la identidad de las personas participantes de los grupos de Facebook utilizados. En este sentido, el estudio tampoco da a conocer citas que posibiliten la identificación de forma indirecta de alguno de sus miembros. Por tanto, el habeas data de estos grupos Facebook, en el marco de la Constitución Política de la República de Chile y la Ley 19.628 sobre protección a la vida privada, cumple con el marco jurídico vigente por cuanto en el acceso a los comentarios y en el tratamiento de los datos sistematizados para este estudio, se respeta plenamente el derecho a la vida privada y el derecho a la protección de los datos personales (Roa, 2013).

\section{Resultados}

Se han considerado como relevantes las formas de aparición del malestar laboral en Facebook, por cuanto esta red social se inscribe al interior de una tendencia nacional y global que comparten usuarios de conferir centralidad en sus vidas a los espacios virtuales como ámbitos de sociabilidad, como también para tramitar el malestar laboral, devenido en un medio fundamental para organizar distintas formas de acción política con potencialidad transformadora (Capogrossi, Magallanes, \& Soraire, 2015; HeiMan, 2008; Sherman, Arriagada, \& Valenzuela,
2013; Valenzuela, 2012, 2013). En este sentido, la interpretación de los hallazgos investigativos se sintetiza en la Figura 1.

\section{Figura 1}

Mapa conceptual de la teorización sobre la influencia del sistema económico neoliberal en el malestar de psicólogos.

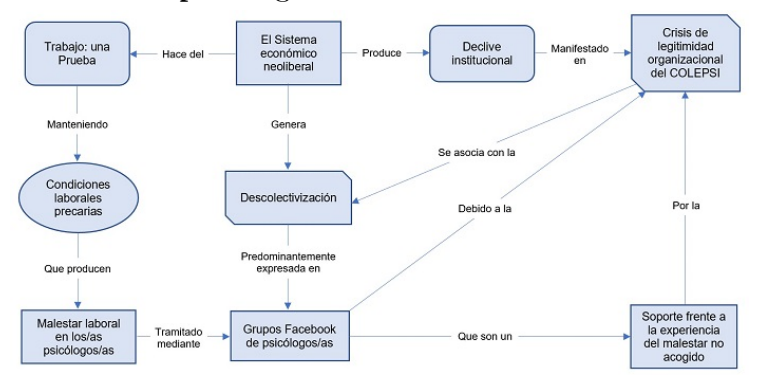

Figura 2

Temas principales comentados en grupos Facebook por psicólogos.

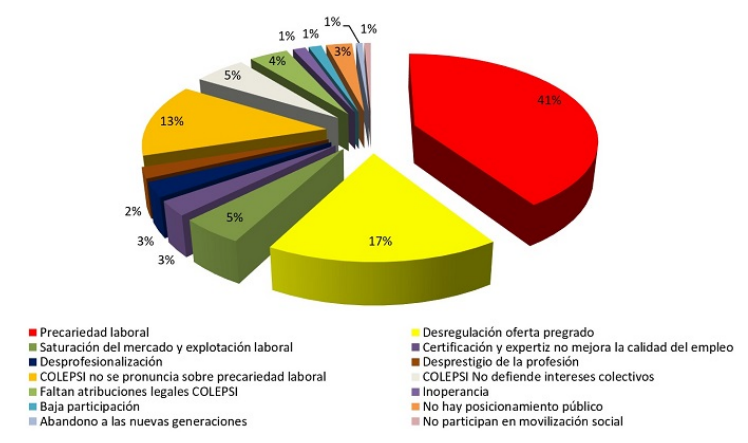

Como resultado del modelo sociopolítico económico neoliberal, los principales aspectos vinculados al malestar laboral (Blanch, 2007; Venegas, 2013) de los psicólogos (Figura 2) se refieren a la "precariedad laboral" (41\%), a la desregulación de la oferta de pregrado (17\%) y una crítica referida al hecho que el COLEPSI no se pronuncia sobre la precariedad laboral (13\%) que experimentan, a lo que se suma la no defensa de intereses colectivos (5\%). Estos resultados dan cuenta de que el neoliberalismo (De Armas $\&$ Venegas, 2015) ha tenido un impacto negativo en la precarización del empleo para los psicólogos (De Armas, Venegas, \& Lorenzo, 2016) y la emergencia del malestar laboral (Blanch, 2007; Venegas, 2013) frente a ello, si bien la crisis de representación del COLEPSI puede asociarse al declive institucional y su consecuencia en la 
descolectivización, la ausencia de comunicados públicos y notorios donde el COLEPSI fije su postura sobre la precariedad laboral, produce una merma en la legitimidad moral (Suchman, 1995) frente a los afiliados y no afiliados. Desde este punto de vista, existiría responsabilidad por parte de los directorios del COLEPSI, particularmente desde el año 2000 en adelante, cuando empieza a consolidarse una desmesurada expansión de la oferta de pregrado y el malestar laboral de los psicólogos que emerge con ello por la precarización del empleo (De Armas et al., 2016).

\section{Figura 3}

Legitimidad pragmática en psicólogos respecto al COLEPSI: porcentaje de afiliados al COLEPSI respecto del total de inscritos en la Superintendencia de Salud a diciembre 2016 según datos de SdeS (2016) y COLEPSI (2016).

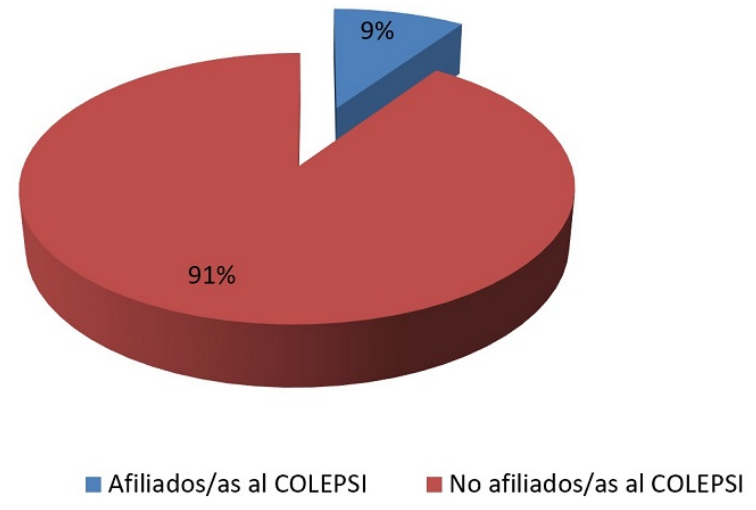

Para inferir la legitimidad pragmática (Suchman, 1995) que tiene para los psicólogos en general el COLEPSI, se dispone que los afiliados a la AG respecto del total de inscritos como prestadores individuales de salud en la Superintendencia de Salud (SdeS), asciende al $9 \%$. Este bajo porcentaje da cuenta de que los psicólogos no encuentran un beneficio personal con el hecho de afiliarse al COLEPSI (Figura 3).

\section{Figura 4}

Temas principales comentados por psicólogos en el grupo Facebook del COLEPSI.

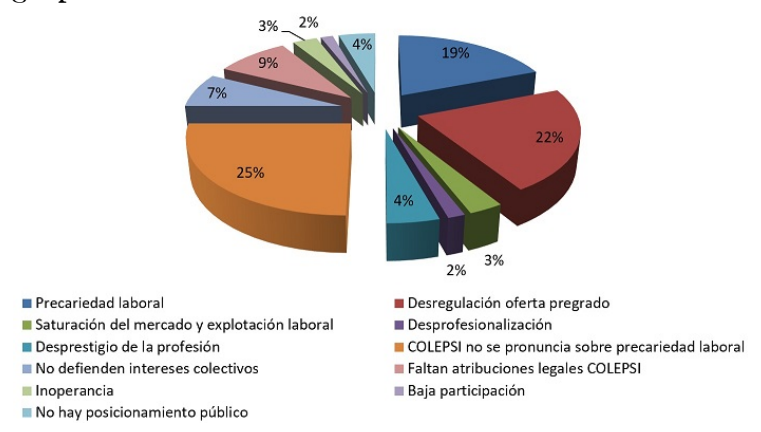

En la Figura 4, se evidencia que frente a la experiencia de "precariedad laboral" (19\%) que a diario viven los psicólogos en Chile "el COLEPSI no se pronuncia sobre esta misma" (25 \%) ni sobre una de las causas que la originaría: "desregulación de la oferta de pregrado" (22 \%). Esto se asocia con la falta de legitimidad moral (Suchman, 1995) del COLEPSI por cuanto los psicólogos afiliados y no afiliados perciben que la AG "no hace lo que debiera", esto es, pronunciarse ante el malestar laboral que les aqueja. Con esta conducta organizacional, el COLEPSI erosionaría la legitimidad "al no mostrar interés" por el malestar laboral, los valores, creencias y objetivos que motivan a los psicólogos. De este modo, se mantiene y profundiza la falta de interés de estos profesionales por afiliarse, y quienes están afiliados no se motivan para mantener al día sus cuotas sociales. Así, se torna más difícil la supervivencia de la organización y la posibilidad de tener los recursos económicos suficientes (Diez et al., 2010) para implementar un cambio organizacional en múltiples niveles, necesario para recuperar legitimidad y un rol protagónico.

Es importante considerar que el modelo neoliberal fomenta una tendencia expansiva de la oferta de pregrado en psicología motivada por el ánimo de lucro, y no considera las reales necesidades del país (De Armas, Ramos, Cárdenas, Ferretti, \& Venegas, 2017; Venegas, 2010), a lo que se debe sumar la falta de atribuciones del Estado a través del Ministerio de Educación para regular la oferta. En un escenario 
como el descrito, la sobreoferta se transforma en una lógica de acumulación capitalista, mediante un "ejército de reserva" de desempleados que presiona a la aceptación y mantención de empleos precarios (Venegas, 2013). página Facebook por los seguidores para emplazar públicamente al COLEPSI como forma de individualización (Robles, 1999) de este malestar laboral (Blanch, 2007; Venegas, 2013).

\section{Figura 5}

Comparación entre grupos Facebook según número de comentarios por tema.

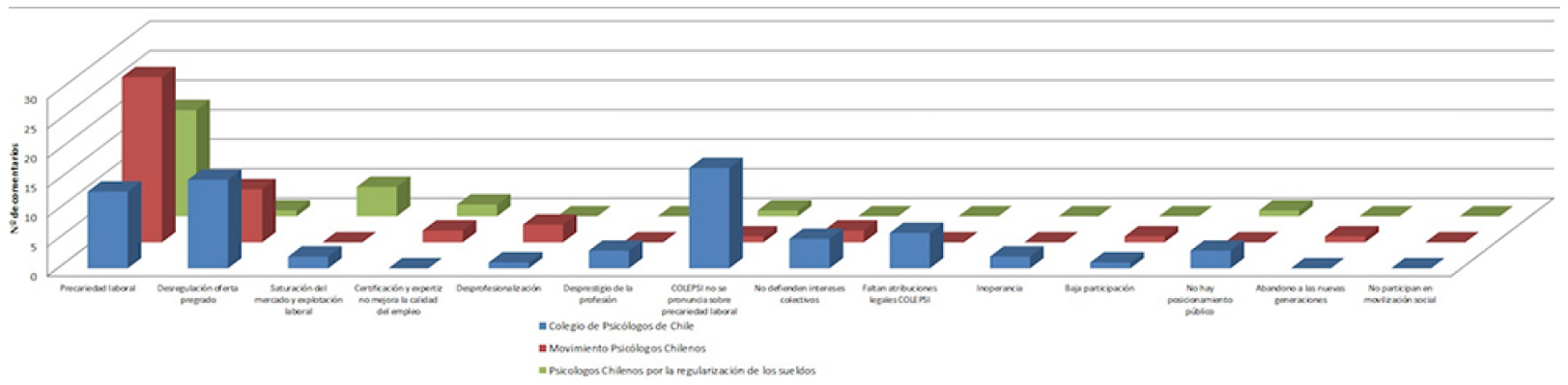

En la página Facebook del COLEPSI, se encuentra que la experiencia de "precariedad laboral” $(F=13)$ vivenciada por psicólogos afiliados y no afiliados no es acogida. Por tanto, se critica a la institución ya que "no se pronuncia sobre la precariedad laboral" $(F=17)$ ni se refiere a la "desregulación de la oferta de pregrado" $(F=$ 15). Si bien se reconoce que existe una "carencia de atribuciones legales en el COLEPSI" $(F=6)$, la crítica también se dirige a que "no defiende intereses colectivos" $(F=5)$. En síntesis, la unidad temática de los comentarios apunta a la falta de legitimidad al colocarse al margen de la experiencia de precariedad laboral de los psicólogos.

De manera global, la temática que mayor interés tiene para los psicólogos en los distintos grupos Facebook son las categorías de precariedadlaboral: "Movimiento de Psicólogos Chilenos" ( $\mathrm{F}=28)$, "Psicólogos Chilenos por la Regulación de los sueldos" ( $\mathrm{F}=$ 18), "COLEPSI" ( $F=13)$ y, por otra parte, la desregulación de la oferta de pregrado "COLEPSI" $(\mathrm{F}=15)$ y "Movimiento de Psicólogos Chilenos" ( $\mathrm{F}=9$ ) (Figura 5). Si se considera el malestar de los psicólogos por la precariedad laboral que viven y la supuesta ausencia de un posicionamiento de la $\mathrm{AG}$, se constata una falta de legitimidad moral (Suchman, 1995). Frente a ello, es utilizada la
El modelo capitalista a través de su discurso de la autonomía individual y la libertad de elección (Ema, 2009), competitividad, autoeficacia y emprendimiento (Laport, Becker, De Sarratea, \& Ocampo, 2010) demandan al COLEPSI, desde la lógica de consumidores que exigen derechos por lo pagado para mantener su afiliación, no como ciudadanos sino como clientes (Moulian, 1997, 1998), este es otro de los efectos del neoliberalismo, modelo que se encuentra estructuralmente limitado para procesar las demandas por derechos sociales, como la calidad del empleo o la sobreoferta de programas de pregrado (Urzúa, Vera-Villarroel, Zúñiga, \& Salas, 2015). Mientras en la lógica individualista tiende a verse en el ejercicio de la profesión más un cumplimiento de un proyecto individual, en contraste con el aporte al país que realiza la profesión desde un marco de compromiso social que el colegio profesional intenta representar en un marco de declive institucional generalizado (Atria, 2015; Atria et al., 2013; Báez, 2017; Dubet, 2006; Garretón, 2016).

\section{Discusión}

El estudio tuvo por objetivo describir la experiencia del malestar laboralnarrada por psicólogos en grupos Facebook relacionados con la formación y ejercicio profesional de la psicología. En el contexto chileno, se detecta un 
trabajo sobre el rol autopercibido del psicólogo (González, González, \& Vicencio, 2014), aunque sería importante proyectar estudios que realicen un análisis íntegro de la salud mental vinculada al trabajo en la profesión. En este sentido, sería importante analizar de qué forma las responsabilidades, el reconocimiento y una mayor variedad en las tareas laborales pueden conducir a un aumento de la satisfacción general en el trabajo en profesionales de la salud mental (Goetz, Kleine-Budde, Bramesfeld, \& Stegbauer, 2018). Si se espera avanzar en mejoras respecto a la calidad laboral, los psicólogos deben avanzar en dos temáticas fundamentales: el bienestar y la equidad, todo lo cual necesita análisis complejos sobre la justicia social y procedimental (Hunter \& Stewart, 2015; Prilleltensky, 2013).

El análisis de la legitimidad organizacional (Suchman, 1995) del COLEPSI, ha sido posible de describir mediante dos dimensiones: legitimidad moral y legitimidad pragmática, sin embargo, los elementos referidos a la legitimidad cognitiva no han podido ser pesquisados, lo que es un desafío en estudios futuros. Sin embargo, se ha logrado comprender la crisis de legitimidad que afecta al COLEPSI, lo que permite explicar en parte el fenómeno de las cuotas sociales o de membresía impagas de sus asociados, como también la baja afiliación de quienes no ven un beneficio para involucrarse en la actividad gremial.

Las altas tasas de desempleo, de necesidades de mayor movilidad laboral, la privatización y reestructuración de las organizaciones laborales (Seršić\& Trkulja, 2009), sumado a los efectos del sistema sociopolítico económico neoliberal han generado una sobreoferta de carreras produciendo una experiencia de malestar laboral en los psicólogos.

Es importante mencionar que el problema enunciado puede tener una multiplicidad de causas y asociarse en algunas aristas en otros lugares del mundo. A modo de ejemplo, en Turquía, la evidencia indica que solamente el $60 \%$ de los graduados en Psicología trabaja en cargos relacionados con la profesión, aunque no existen datos sobre la empleabilidad y la distribución de los graduados en psicología y tampoco sobre su satisfacción laboral (Sumer, Helvaci, \& Misirlisoy, 2013). Por su parte, en Colombia, se llevó a cabo una caracterización de la empleabilidad de psicólogos, a través de un estudio de caso en una institución de educación superior, evidenciando la necesidad de realizar investigaciones que permitan diferenciar entre la inserción laboral y la empleabilidad, las acciones que las asociaciones gremiales, las organizaciones y los Estados promueven para mejorar y potenciar las condiciones profesionales del ejercicio de la disciplina (Aguilar-Bustamante, 2011).

Es importante destacar que entre las limitantes de la presente investigación está el que no fue posible conocer las diferencias existentes entre psicólogos que llevan una carrera temprana, mitad de carrera y final de carrera (Dorociak, Rupert, \& Zahniser, 2017), tampoco se analizan variables como el costo de la educación versus el potencial de ingresos (Doran, Marks, Kraha, Ameen, \& El-Ghoroury, 2016) o las complejidades de la relación familia-trabajo (Rupert, Stevanovic, Tuminello Hartman, Brayant, \& Miller, 2012).

Finalmente, se sugiere replicar este tipo de estudios no solo en Chile, sino en diversos países de América Latina para tener un mapa más íntegro de cómo afecta el malestar laboral la profesión de psicólogo.

\section{Referencias}

Acuña, P. (2017). Discurso del presidente electo del Colegio de Psicólogos de Chile con motivo del 49.. aniversario del Colegio de la Orden. Recuperado de http://colegiopsicologos.cl/2017/12/12/p alabras-del-presidente-electo/discurso-s-pe dro-acuna/

Aguilar-Bustamante, $\quad$ M. C. (2011).Caracterización de la empleabilidad de los egresados de la Universidad Santo Tomás, a la luz del Modelo Forrier y Sels (Tesis de maestría inédita). Universidad Católica de Colombia, Bogotá.

Aguilar-Bustamante, M. C. (2017).Estudio comparativo del estilo de liderazgo y la cultura 
organizacional en asociaciones gremiales $y$ directores de programas en Psicología en Latinoamerica y España, con base en el proyecto Globe (Tesis de doctorado inédita). Universidad de La Laguna, Tenerife, España. Recuperado de https://dialnet.unir ioja.es/servlet/tesis? codigo $=158135$

Alonso, L. (1998). La mirada cualitativa en sociología: una aproximación interpretativa. Madrid: Fundamentos.

Andrade, V. (2014). Identidad profesional y el mundo del trabajo contemporáneo. Reflexiones desde un resumen de caso. Athenea Digital, 14(2), 117-145. https://doi .org/10.5565/rev/athenea.1143

Araujo, K. (2009). Individuo y feminismo. Notas desde América Latina. Íconos, 33, 141-153. https://doi.org/10.17141/iconos.3 3.2009.307

Araujo, K., \& Martuccelli, D. (2012a). Desafíos comunes. Retrato de la sociedad chilena y sus individuos (Vol. 1). Santiago: Lom.

Araujo, K., \& Martuccelli, D. (2012b). Desafios comunes. Retrato de la sociedad chilena y sus individuos (Vol. 2). Santiago: Lom.

Asún, D. (2002). Dimensiones psicosociales de las minorías activas. En F. Morales, D. Páez, A. Kornsblit \& D. Asún (Eds.), Psicología social (pp. 333-358). Buenos Aires: Prentice Hall.

Atria, F. (2015). La Constitución tramposa (4. ed.). Santiago: Lom.

Atria, F., Larraín, G., Benavente, J., Couso, J., \& Joignant, A. (2013). El otro modelo. Del orden neoliberal al régimen de lo público. Barcelona: Debate.

Báez, F. (2017). Diseño institucional y neoliberalismo. El modelo chileno como resultado del quiebre unilateral del contrato social. Papers, 102 (3), 449-476. https://doi. org/10.5565/rev/papers.2299

Blanch, T. (2007). Psicología social del trabajo En M. Aguilar \& A. Reid (Coords.), Tratado de psicología social. Perspectivas socioculturales(pp. 210238). México: Anthropos.

Cáceres, P. (2008). Análisis cualitativo de contenido: una alternativa metodológica alcanzable. Psicoperspectivas, 2, 53-82. https://doi.org/10.5027/psicopers pectivas-Vol2-Issue1-fulltext-3

Cahís, F. (27 de diciembre de 2016). Psicología en Chile: publicidad engañosa.Recuperado de http://radio.uchile.cl/2016/12/27/psicolo gia-en-chile-publicidad-enganosa/

Capogrossi, M., Magallanes, M., \& Soraire, F. (2015). Los desafíos de Facebook. Apuntes para el abordaje de las redes sociales como fuente. Revista de Antropología Experimental, 15, 47-63. https://doi.org/10.17561/rae.v0i 15.2390

Castel, R. (1997). La metamorfosis de la cuestión social. Una crónica del salariado. Buenos Aires: Paidós.

Castel, R. (2004). La inseguridad social: ¿Qué es estar protegido? Buenos Aires: Manantial.

Colegio de Psicólogos de Chile. (2018). Objetivos del Colegio de Psicólogos de Chile (AG). Recuperado de http://colegiopsicologos.cl/ nosotros/objetivos/

Dattilio, F. M. (2015). The self-care of psychologists and mental health professionals: A review and practitioner guide. Australian Psychologist, 50(6), 393-399. https://doi.org/10.1111/ap.12157

Dávila, O. (1998). Estado y políticas sociales. Del Estado protector al Estado subsidiario. Última Década, 9, 1-9. Recuperado de https://www.redalyc.org/comocitar.oa?i $\mathrm{d}=19500906$

De Armas, T., Ramos, M., Cárdenas, T., Ferretti, P., \& Venegas, C. (2017). La acreditación de la Educación Superior en Chile: el ocaso de la psicología con compromiso social. Alternativas Cubanas de Psicología, 15(5), 87-108. Recuperado de http://www.alfepsi.org/wp-content/uplo ads/2017/08/alternativas-cubanas-en-psico logia-v5n15.pdf

De Armas, T., Ramos, M., \& Venegas, C. (2016a). La psicología en Chile: desideologizando la formación de pregrado y el ejercicio profesional. Integración Académica de la Psicología, 11(4), 28-48. Recuperado de http://integracion-academica.org/comp 
onent/content/article/20-volumen-4-nume ro-11-2016/127-la-psicologia-en-chile-desi deologizando-la-formacion-de-pregrado-yelejercicio-profesional

De Armas, T., Ramos, M., \& Venegas, C. (septiembre, 2016b). El Colegio de Psicólogos de Chile: sociedad contemporánea y profesión. En Hacer y Pensar la Psicología (Vol. I). Memorias del V Congreso Alfepsi (pp. 75-86). San José de Costa Rica: Asociación Latinoamericana para la Formación y Enseñanza de la Psicología.

De Armas, T., \& Venegas, C. (octubre, 2015). El diseño socio-político institucional y la psicología como profesión en Cuba y Chile: un análisis crítico. En R. Jaimes (Ed.), Memorias IV Congreso Alfepsi, Identidad, Innovación y Compromiso Social (pp. 1247-1260). Santa Marta, CO: Asociación Latinoamericana para la Formación y Enseñanza de la Psicología.

De Armas, T., Venegas, C., \& Lorenzo, A. (2016). La psicología en el Chile postdictatorial: hacia la (des)profesionalización. Alternativas Cubanas de Psicología, 11(4), 27-46. Recuperado de http://acupsi.org/articulo/1 51/la-psicologa-en-el-chile-postdictatorialhacia-la-desprofesionalizacin.html

De Souza, A. A., \& De la Coleta, M. F. (2015). Professional profile, well-being and job satisfaction among psychologist working in public healthcare services. Estudos de Psicologia, 32 (2), 249-258. https://doi.org/1 0.1590/0103-166X2015000200009

Diez, F., Blanco, A., \& Prado, C. (2010). La legitimidad como factor del éxito organizativo. Investigaciones Europeas de Dirección y Economía de la Empresa, 16(3), 127-143. Recuperado de https://ideas.repec .org/a/idi/jiedee/v16y2010i3p127-143.html

Doran, J. M., Marks, L. R., Kraha, A., Ameen, E. J., \& El-Ghoroury, N. H. (2016). Graduate debt in psychology: A qualitative analysis. Training and Education in Professional Psychology, 10(4), 179-187. h ttps://doi.org/10.1037/tep0000132
Dubar, C. (2001). El trabajo y las identidades profesionales y personales. Revista Latinoamericana de Estudios del Trabajo, 13, 5-16. Recuperado de https://vdocuments.site/dubar-2001-el-t rabajo-y-las-identidades-profesionales-y-pe rsonales.html

Dubar, C. (2002). La crisis de las identidades. La interpretación de una mutación. Barcelona: Bellaterra.

Dubet, F. (2006). El declive de la institución: profesiones, sujetos e individuos en la modernidad. Barcelona: Gedisa.

Dorociak, K. E., Rupert, P. A., \& Zahniser, E. (2017). Work life, well-being, and self-care across the professional lifespan of psychologists. Professional Psychology: Research and Practice, 48(6), 429-437. https ://doi.org/10.1037/pro0000160

Ema, J. (2009). Capitalismo y subjetividad. ¿Qué sujeto, qué vínculo y qué libertad? Psicoperspectivas, 8(2), 224-247. Recuperado desde http://www.psicoperspec tivas.cl/index.php/psicoperspectivas/article /view/61

Firat, M. (2017). Relationship between selfcontrol and Facebook use: Case of CEIT Students. Kuram ve Uygulamada Egitim Bilimleri, 17(4), 1179-1201.

Flick, U. (2004). Introducción a la investigación cualitativa. Madrid: Morata.

Flick, U. (2015). El diseño de investigación cualitativa. Madrid: Morata.

Forrier, A., \& Sels, L. (2003). The concept employability: A complex mosaic. International Journal of Human Resources Development and Management, 3,102-124. Recuperado de http://www.ouderenenarbei d.be/documenten/artikel\%20ijhrdm.pdf

Friedman, M., \& Friedman, R. (1986). Libertad de elegir. Madrid: Orbis.

Gamboa, J., Gracia, F., Ripoll, P., \& Peiró, J. M. (2007). La empleabilidad y la iniciativa personal como antecedentes de la satisfacción laboral. Valencia: Instituto Valenciano de Investigaciones Económicas. Recuperado de http://www.ivie.es/downloads/docs/wpas ec/wpasec-2007-01.pdf 
Garretón, M. (2016). La ruptura entre política y sociedad. Una introducción. En M. Garretón (Coord.), La gran ruptura. Institucionalidad política y actores sociales en el Chile del Siglo XXI. Santiago: Lom.

Gibbs, G. (2012). El análisis de datos cualitativos en investigación cualitativa. Madrid: Morata.

Goetz, K., Kleine-Budde, K., Bramesfeld, A., \& Stegbauer, C. (2018). Working atmosphere, job satisfaction and individual characteristics of community mental health professionals in integrated care. Health in Social Care in the Community, 26(2), 176-181. https://doi.org/10.1111/hsc.1249 9

Gómez, J. (2010). Política, democracia y ciudadanía en una sociedad neoliberal. Chile: 1990-2010). Santiago: Arcis.

González, M., González, I., \& Vicencio, K. (2014). Descripción sobre el rol autopercibido del psicólogo y sus implicancias en los procesos de formación de pregrado. Psicoperspectivas, 13(1), 108-120. https://doi.org/10.5027/psicopers pectivas-vol13-issue1-fulltext-296

Gutiérrez, A., \& Palacios, B. (2016). Análisis de datos cualitativos con QRS NVivo 11. Madrid: Editorial Fundación iS $+\mathrm{D}$ para la Investigación Social Avanzada.

Hei-Man, T. (2008). Ethnography of social network in cyberspace: The Facebook phenomenon. The Hong Kong Anthropologist, 2, 53-77. Recuperado de htt p://citeseerx.ist.psu.edu/viewdoc/download ?doi $=10 \cdot 1 \cdot 1 \cdot 470.3991 \&$ rep $=$ rep $1 \&$ type $=$ pdf

Heritage Foundation. (2013). About the index. Recuperado de http://www.heritage.org/ind ex/about

Hernández, R., Fernández, C., \& Baptista, M. (2010). Metodología de la investigación (5. ed.). México: McGraw-Hill.

Hillage, J., \& Pollard, E. (1998). Employability: Developing a framework for policy analysis. Londres: Department for Education and Employment.

Hunter, A. G., \& Stewart, A. J. (2015). Past as prologue: How history becomes psychologically present. Journal of Social Issues, 71(2), 219-228. https://doi.org/10.11 11/josi.12106

Idsoe, T. (2006). Job aspects in the School Psychology Service: Empirically distinct associations with positive challenge at work, perceived control at work, and job attitudes. European Journal of Work and Organizational Psychology, 15(1), 46-72. htt ps://doi.org/10.1080/13594320500411514

Johnson, W. B., Johnson, S. J., Sullivan, G. R., Bongar, B., Miller, L., \& Sammons, M. T. (2011). Psychology in extremis: Preventing problems of professional competence in dangerous practice settings. Professional Psychology: Research and Practice, 42(1), 94-104. https://doi.org/10.1037/a0022365

Kaiser, A. (2017). La tiranía de la igualdad. ¿Por qué el igualitarismo es inmoral y socava el progreso de nuestra sociedad? Barcelona: Ediciones Deusto.

Laport, N., Becker, A., De Sarratea, A., \& Ocampo, G. (2010). Vinculación laboral flexible: construcción de identidad laboral en psicólogos adultos jóvenes. Psicoperspectivas, 9(1), 138-157. https://doi.org/10.5027/psicopers pectivas-Vol9-Issue1-fulltext-91

Maeran, R., Robusto, E., \& Anselmi, P. (2015). Agora\# Project: Job placement, skills assessment and job satisfaction of graduates in Psychology at the University of Padua. Giornale Italiano di Psicologia, 42 (4), 755-776. https://doi.org/10.1421/81941

Malinowski, A. J. (2013). Characteristics of job burnout and humor among psychotherapists. Humor, 26(1), 117-133. h ttps://doi.org/10.1515/humor-2013-0007

Martuccelli, D. (2007a). Gramáticas del individuo. Buenos Aires: Losada.

Martuccelli, D. (2007b). Cambio de rumbo. La sociedad a escala del individuo. Santiago: Lom.

Martuccelli, D. (2010a). La individuación como macrosociología de la sociedad singularista. Persona y Sociedad, 24(3), 9-29. Recuperado de http://repositorio.uahurtado.cl/handle/1 $1242 / 3854$ 
Martuccelli, D. (2010b). La sociología en tiempos del individuo. Entrevista de R. Martinic y N. Soto. Doble Vínculo, 1, 1-26. Recuperado de https://doblevinculo.files.wordpress.com /2011/01/entrevista-a-danilo-martuccelli.p $\mathrm{df}$

Melús, A. (diciembre, 2016). Discurso con motivo de la Ceremonia Aniversario 48. ㅁ del Colegio de Psicólogos de Chile. Santiago de Chile. Recuperado de http://colegiopsicologos.cl/ wp-content/uploads/2016/12/discurso-aniv ersario-2016.pdf

Melús, A. (julio, 2017). Discurso de la presidenta del Colegio de Psicólogos de Chile con motivo de la inauguración del VIII Congreso Nacional de Psicología. Santiago de Chile. Recuperado de http://colegiopsicologos.cl/wp-content/u ploads/2017/07/Discurso-Ps.-Alejandra-M el\%C3\%BAs.pdf

Moscovici, S. (1985). Psicología social (Vol. 1). Barcelona: Paidós.

Moulian, T. (1997). Chile actual anatomía de un mito. Santiago: Lom.

Moulian, T. (1998). El consume me consume. Santiago: Lom.

Mustaffa, C. S., \& Ibrahim, N. (2014). Perception and the use of social media from the perspective of parents: A qualitative analysis [Edición especial].Journal Komunikasi: Malasyan Journal of Communication, 30, 43-74. https: //doi.org/10.17576/JKMJC-2014-30SI-04

Peiró, J. M., Ayala, Y., Tordera, N., Lorente, L., \& Rodríguez, I. (2014). Sustainable wellness at work: Review and reformulation. Papeles del Psicólogo, 35(1), 5-14. Recuperado de https:/www.redalyc.org/comocitar.oa?i $\mathrm{d}=77830184002$

Prilleltensky, I. (2013). Wellness without fairness: The missing link in psychology.South African Journal of Psychology, 43(2), 147-155. https://doi.org/10.1177/00812463 13484238

Proserpio, D., Counts, S., \& Jain, A. (mayo, 2016). The psychology of job loss: Using social media data to characterize and predict unemployment. WEbSci'16 Proceedings of The 8th International ACM
Web Science Conference 2016 (pp.223-232). Nueva York: Association for Computing Machinery https://doi.org/10.1145/290813 1.2913008

QSR International. (2017). NVivo (Versión 11). [Software]. Melbourne: QSR International.

QSR International. (2018). How are cluster analysis diagrams generated? Recuperado de http://helpnv11.qsrinternational.com/d esktop/deep_concepts/how_are_cluster_an alysis_diagrams_generated_htm

Ramadan, Z. (2017). Examining the dilution of the consumer-brand relationship on Facebook: The saturation issue. Qualitative Market Research, 20(3), 335-353. https://do i.org/10.1108/QMR-07-2016-0064

Rentería-Pérez, E., \& Malvezzi, S. (2008). Empleabilidad, cambios y exigencias psicosociales en el trabajo. Universitas Psychologica, 7(2), 319-334. Recuperado de http://pepsic.bvsalud.org/pdf/up/v7n2/v 7n2a02.pdf

Roa, M. (2013). Facebook frente al derecho a la vida privada y la protección de datos personales (Tesis de licenciatura inédita). Universidad de Chile, Santiago. Recuperado de http://repositorio.uchile.cl/bitstream/ha ndle/2250/113249/de-roa_m.pdf?sequence $=1$ \&isAllowed $=\mathrm{y}$

Robles, F. (1999). Los sujetos y la cotidianidad. Elementos para una microsociología de lo cotidiano. Talcahuano: Sociedad Hoy.

Roncalli, S., \& Byrne, M. (2016). Relationships at work, burnout and job satisfaction: A study on Irish psychologists. Mental Health Review Journal, 21 (1), 23-36. https://doi.org /10.1108/MHRJ-01-2015-0002

Rupert, P. A., Stevanovic, P., Tuminello Hartman, E. R., Brayant, F. B., \& Miller, A. (2012). Predicting work-family conflict and life satisfaction among professional psychologists. Professional Psychology: Research and Practice, 43(4), 341-348. https://doi.org/10.1037/a0026675

Salas, G., \& Lizama, E. (2013). Historia de la psicología en Chile. 1889-1981 (2. ${ }^{a}$ ed.). La Serena: Editorial Universidad de La Serena. 
Salinas, D. (2010). ¿A cuántos y a quiénes preguntar? Una aproximación al muestreo cuantitativo y cualitativo en investigación social y educacional. Valparaíso: Ediciones Universitarias de la Pontificia Universidad Católica de Valparaíso.

Sagun, K., \& Luyt, B. (2017). Cultured and popular literary circuits on Facebook: A case study of Singaporean print culture in social media. Information Research, 22(2), paper 746. Recuperado de http://Informati onR.net/ir/22-21/paper746.html

Sauter, S. L., \& Hurrell, J. J. (2017). Occupational health contributions to the development and promise of occupational health psychology. Journal of Occupational Health Psychology, 22(3), 251-258. https://d oi.org/10.1037/ocp0000088

Seršić, D. M.,\& Trkulja, J. (2009). Job insecurity as a research subject in psychology: Theories, definitions, findings. Drustvena Istrazivanja, 18(3), 523-545. Recuperado de https://scholar.google.hr/scholar?=en\& $\mathrm{q}=$ Nesigurnost $\% 20$ posla $\% 20 \mathrm{kao} \% 20$ prem et $\% 20$ istra\%C5\%BEivanja $\% 20$ u $\% 20$ psihol ogiji:\%20metode\%20i\%20rezultati\&btnG $=$

Sharrocks, L. (2014). School staff perceptions of well-being and experience of an intervention to promote well-being. Educational Psychology in Practice, 30(1), 19-36. https://doi.org/10.1080/02667363.2 013.868787

Sherman, A., Arriagada, A., \& Valenzuela, S. (2013). La protesta en la era de las redes sociales: el caso chileno. En A. Arriagada \& P. Navia (Eds.), Intermedios: medios y democracia en Chile (pp. 181-199). Santiago: Editorial Universidad Diego Portales.

Soto, A. (2011). Narrativas de profesionales chilenos sobre sus trayectorias laborales: la construcción de las identidades en el trabajo. Psykhe, 20(1), 15-27. https://doi.or g/10.4067/S0718-22282011000100002

Suchman, M. (1995). Managing legitimacy: Strategic and institutional approaches. Academy of Management Review, 20(3),
571-610. https://doi.org/10.5465/amr.1995 .9508080331

Sumer, N., Helvaci, E., \& Misirlisoy, M. (2013). Employability of psychology graduates and their job satisfaction in Turkey: An online survey. Psychology, Learning and Teaching, 12(2), 189-195. https://doi.org/10.2304/pla t.2013.12.2.189

Superintendencia de Salud. (2016). Estadística de prestadores individuales de Salud. Período 1. - de abril 2009 al 31 de diciembre 2016. Recuperado de https://www.supersalud.gob.cl/documen tacion/666/articles-14696_recurso_1.pdf

Stake, R. (1999). Investigación con estudio de casos. Madrid: Morata.

Urzúa, A., Vera-Villarroel, P., Zúñiga, C., \& Salas, G. (2015). Psicología en Chile. Análisis de su historia, presente y futuro.Universitas Psychologica, 14(3), 1125-1142. https://doi. org/10.11144/Javeriana.upsy14-3.pcah

Valenzuela, S. (2012). La protesta en la era de Facebook: manifestaciones juveniles y uso de redes sociales en Chile 2009-2011. En A. Sherman (Ed.), Jóvenes, participación y medios 2011 (pp. 20-29). Santiago: Ediciones Universidad Diego Portales.

Valenzuela, S. (2013). Unpacking the use of social media for protest behavior: The roles of information, opinion expression, and activism. American Behavioral Scientist, 57(7), 920-942. https://journals.sagepub.co $\mathrm{m} /$ doi $/ 10.1177 / 0002764213479375$

Venegas, C. (2010). La realidad laboral de la psicología en Chile y la V Región: iLibre mercado o dignidad humana? Razón y Palabra, 72, 1-56. Recuperado de http://www.razonypalabra.org.mx/N/N7 2/Varia_72/19_Venegas_72.pdf

Venegas, C. (2013). Capitalismo y subjetividad laboral. El discurso del malestar docente. Aposta Digital, 59, 1-53. Recuperado de http://apostadigital.com/revistav3/heme roteca/cvenegas2.pdf

Venegas, C. (2014). La psicología en Chile: Aportes para profundizar la regulación ética de la profesión. En C. Venegas (Ed.), La formación por competencias en psicología. 
Análisis crítico y propuesta de un taller para el desarrollo valórico-actitudinal(pp. 17-30). Viña del Mar: Editorial Altazor.

Venegas, C. (2019a). Formación de pregrado y ejercicio profesional de la Psicología en Cuba y Chile desde una teoría de individuación (Tesis de doctorado inédita). Santiago, Universidad de Arte y Ciencias Sociales.

Venegas, C. (2019b). Políticas de formación y condiciones laborales. Manuscrito inédito, Colegio de Psicólogos de Chile, Santiago de Chile.

\section{Notas}

* Artículo de investigación.

1 Si bien se concuerda con la noción lingüística y de género que refiere una distinción gramatical entre psicólogos y psicólogas, por cuestiones de parsimonia y estética se hará solamente alusión a psicólogos. La misma situación aplica a conceptos como afiliados y/o trabajadores. Se espera esta aclaración sirva de disculpas a quien no comprenda las razones.

2 Este aspecto es tan importante que el actual Directorio Nacional encabezado por Pedro Acuña Mercier (2018-2020) ha impulsado una "política permanente de moratoria", por medio de la cual los afiliados que tengan más de un año de retraso en sus cuotas sociales o de membresía solo deben pagar un año y quedan al día. En caso de tener dificultades económicas, habrá facilidades de pago. De lo contrario, se debe proceder a su desafiliación aplicando el reglamento vigente.

3 Actualmente existen 10 filiales activas.

4 Anterior al año 2012, la distribución de las cuotas sociales o de membresía era así: 50 \% quedaban a disposición de la respectiva filial regional y el otro $50 \%$ estaba destinado a la administración del Directorio Nacional del COLEPSI.

5 Rodrigo Molina Miranda, además de ser expresidente del COLEPSI, es secretario en el actual Directorio Nacional del COLEPSI, encabezado por Pedro Acuña Mercier (2018-2020).

6 El COLEPSI se encuentra federado en la Federación de Colegios Profesionales Universitarios de Chile (FEDECOL), actualmente integrada por 17 AG que reconocen el interés transversal de las profesiones, en términos de recuperar la jurisdicción ética, reivindicar la dignidad de las profesiones e impulsar el Proyecto de Ley de Colegios Profesionales aún en trámite desde el año 2009 en el Congreso Nacional de Chile.

7 Por el contrario, los "grupos cerrados" con un elevado número de seguidores no pueden ser cambiados por los administradores de los respectivos grupos a la condición de "grupos abiertos" por las restricciones actuales de las políticas de Facebook. Dentro de los "grupos cerrados" se encontraba la Asociación de Psicólogos Chilenos y Psicólogos Chilenos que no pudieron ser considerados para el estudio por cuanto el software NVivo 11 viene configurado solo para extraer información desde "grupos públicos". 